\title{
Author Correction: Atmospheric mountain wave generation on Venus and its influence on the solid planet's rotation rate
}

\author{
T. Navarro, G. Schubert and S. Lebonnois (D)
}

Correction to: Nature Geoscience https://doi.org/10.1038/s41561-018-0157-x, published online 18 June 2018.

In the version of this Article originally published, a statement regarding past measurements of the length of day and rotation rate of Venus was potentially misleading. The original statement has now been replaced in the online versions of this Article, to acknowledge that neither Magellan nor Venus Express measured an instantaneous rotation rate. The original and corrected statements are shown below.

\section{Original}

Sixteen years apart, the Magellan and Venus Express missions measured a length of day (LOD) difference of 7 minutes ${ }^{14}$. This longterm trend can be partially explained by the intradiurnal change of LOD due to atmospheric motions.

\section{Corrected}

An estimate of the Venusian length of day (LOD) was obtained by averaging 500 days of radar surface images taken by the Magellan mission. Another estimate was also obtained by comparing the relative change of position of topographical features seen by Magellan and Venus Express 16 years apart, resulting in a difference of 7 minutes of LOD for these two timespans ${ }^{14}$. This difference can be partially explained by an intradiurnal change of LOD due to atmospheric motions, although systematic errors may also contribute.

\section{Author Correction: A changeable day in the life of Venus}

James Tuttle Keane

Correction to: Nature Geoscience https://doi.org/10.1038/s41561-018-0179-4, published online 18 June 2018.

In the version of this Sketch-Up originally published, labels describing atmospheric torques acting on Venus were incorrect. The sketch suggested that the speed-up effect of mountain waves is balanced by a slow-down from thermal tides; instead, both mountain waves and thermal tides speed up the rotation, and this effect is balanced by a slow-down from the solar gravitational torque on the solid body, which is transmitted to the atmosphere. This error has now been corrected in the online versions by adjusting the relevant labels; that is, "Mountain waves speed the planet up" has been changed to "Mountain waves and thermal tides speed the planet up", and "Thermal tide slows the planet down" has been changed to "Solar gravitational torques slow the planet down".

\section{Author Correction: Microbial life and biogeochemical cycling on land 3,220 million years ago}

Martin Homann, Pierre Sansjofre, Mark Van Zuilen, Christoph Heubeck, Jian Gong, Bryan Killingsworth (iD, Ian S. Foster (D), Alessandro Airo, Martin J. Van Kranendonk (D), Magali Ader and Stefan V. Lalonde (D)

Correction to: Nature Geoscience https://doi.org/10.1038/s41561-018-0190-9, published online 23 July 2018.

In the version of this Article originally published, author Magali Ader was wrongly linked to affiliation 3; it should have been affiliation

2. This error has now been corrected in the online versions. 